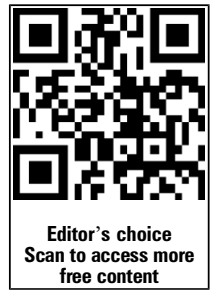

free content
${ }^{1}$ Department of Palliative Care, Weston Area Health Trust, Weston super Mare, UK ${ }^{2}$ Department of End of Life Care, University of Bradford, Bradford, UK

\section{Correspondence to} Dr Julian Abel, Department of Palliative Care, Weston Area Health Trust, Weston Area Health Trust, Grange Rd, Weston super Mare, BS23 4TQ, UK; julian.abel@nhs.net

Received 21 August 2015 Revised 18 December 2015 Accepted 10 January 2016 Published Online First 31 January 2016
CrossMark

To cite: Abel J, Kellehear A. BMJ Supportive \& Palliative Care 2016;6:21-26.

\title{
Palliative care reimagined: a needed
} shift

\author{
Julian Abel, ${ }^{1}$ Allan Kellehear ${ }^{2}$
}

\section{ABSTRACT}

Palliative care, since its inception over 60 years ago, has set the standard of how to care for people who are dying. Key features among these standards have been the professional development of clinical specialisms such as palliative medicine and palliative nursing; the essential addition of the multidisciplinary team to these two new specialisms that included social, spiritual and allied health workers-an outgrowth of the recognition that routine work with the dying, their carers, and the bereaved required more than solely clinical skills; and the unique partnership with communities that yielded the volunteer movement within palliative care. Professional, evidence-based symptom management and the importance of supportive care in its widest possible sense were and remain the cornerstones of the modern palliative care approach. However, the majority of people with terminal illnesses do not have access to palliative care teams, whose main focus of care remains patients with cancer. In the context outlined above this paper therefore poses two key questions: how can we provide an equitable level of care for all people irrespective of diagnosis and how can we increase the range and quality of non-medical/nursing supportive care in a context of diminishing resources? We argue that an important opportunity and solution can be found by adopting the principles of a public health approach to end-of-life care.

Palliative care, since the formation of $\mathrm{St}$ Christopher's Hospice in 1967 has set the standard of how to care for people who are dying. Key features among these standards have been the professional development of clinical specialisms such as palliative medicine and palliative nursing; the essential addition of the multidisciplinary team to these two new specialisms that included social, spiritual and allied health workers-an outgrowth of the recognition that routine work with the dying, their carers and the bereaved required more than solely clinical skills; and the unique partnership with communities that yielded the volunteer movement within palliative care. Professional, evidence-based symptom management and the importance of supportive care in its widest possible sense were and remain the cornerstones of the modern palliative care approach.

While most people will agree that these developments have been outstanding, yet some long-term problems remain while other recent national developments threaten early achievements. Among these current challenges are the following:

1. The home death rate, over $30 \%$ in the 1980s in Britain, has remained low, with rates being near 20\%. The National End-of-Life Care Strategy was published in the UK in 2008 with the specific purpose of resolving some of these problems. Despite the enormous effort that has taken place, the increase in home death rates is disappointingly modest, being $22.6 \%$ in $2014^{1}$

2. Both primary and secondary care struggle to identify people who are at the last phase of their life and consequently there is limited uptake of advance care planning. Reporting data from electronic palliative care coordination systems show that people with non-cancer diagnoses have hospital death rates of between 10 and $20 \%$ when offered advance care planning, with their choices are available to the broader health community electronically. ${ }^{2}$ The implication is that with greater uptake of the principles of end-of-life care, the greater the impact on quality of care and place of death.

3. Availability of professional caring services in the home to look after people with terminal illnesses is diminishing. In 2014, 9 of 10 local authorities reported that they were limiting social care packages to those people who are in crisis. ${ }^{3}$ Budgets for social services have been reduced by as much as $40 \%$. Some of the improvements 
we have seen in trends for place of death are levelling out and in some places worsening.

4. Specialist palliative care, by and large, remains firmly for patients with cancer. This is particularly the case outside of hospital care. The challenge of how to provide specialist palliative care principles for all patients without cancer remains unanswered. The demographic and epidemiological challenges that loom large for palliative care remain the older population with multiple morbidities, people in remote and rural areas, the disabled, imprisoned, those in nursing and care homes, and the homeless. Continuity of care remains a serious challenge and with diminishing social care resources this problem will only become greater. Palliative care is not only about maximising support for dying patients and their families and also the bereaved. Identifying and deploying social care resources for support outside episodes of medical and nursing care and beyond the death of the patient (for support of the bereaved) has always been a difficult ask but in the current context of shrinking budgets will now also be an impossible one.

In the context outlined above this paper therefore poses two key questions: how can we provide an equitable level of care for all people irrespective of diagnosis and how can we increase the range and quality of non-medical/nursing supportive care in a context of diminishing resources? We argue that an important opportunity and solution can be found by adopting the principles of a public health approach to end-of-life care.

\section{WHAT IS PUBLIC HEALTH}

At the core of all of our historical public health efforts in all the major health and medical fields is a blended approach of 'top-down' efforts such as direct service provision, legislative changes and health surveillance combined with 'bottom-up' efforts such as local community participation and actions, and the promotion of individual responsibility. Although all public health traditions-both imposed and participatory-have been at the heart of all major efforts at prevention and harm-reduction, it has been the local community participation and action, and promoting individual responsibility approaches, that have been crucial to increasing access and quality, ensuring success, and guaranteeing the sustainability of most public health programmes. Moreover, it has been the bottom up approaches in public health (aka health promotion and community development) that have provided alternatives to direct services provision by exploiting the social assets (local social, financial, practical supports) of a community while ensuring continuity of care that paid service provision simply cannot provide without inconceivable injections of government or private funding.

In summary, all successful national public health programmes involve an educational component, a community effort, and a government/services role.
The aim of a well-rounded public health strategy then is to recognise the necessary limits to government/ service provision. In this context, we then: (1) promote community development and engagement, working to fill the support spaces outside episodes of professional care and; (2) develop those spaces and times outside service provision as key fertile ground for the planning, development and uptake of our efforts in early intervention. This approach to community participation serves to promote and nurture the social capital (trust, empathy and cooperation) in every community in the service of prevention, harmreduction and early intervention for illnesses and other harms but also more positively, health promotion, education and well-being.

\section{A PUBLIC HEALTH APPROACH TO END-OF-LIFE CARE}

A stark reality for end-of-life care has been the lack of equitable provision of specialist palliative care to people who have terminal illnesses other than cancer. Since the publication of the National End-of-Life Strategy in 2008, with the formation of the National End-of-Life Care Programme led by Professor Sir Mike Richards, extensive investment and initiatives have taken place across the UK to improve end-of-life care in all settings. While there have been many improvements, the widespread application and uptake of advance care planning has been sporadic. Changes in home death rates have been very modest in the light of this sustained effort. Hospital death rates for non-cancer terminal diagnoses remain high, with average hospital cancer death rates being $37.82 \%$ and non-cancer diagnoses between 48 and 63\% depending on the condition. ${ }^{4}$ Given the current demands on professional and social care, there is little realistic prospect of meeting an increased demand using professional services should more people be offered advance care planning and choose a home death. We propose a new way of restructuring palliative care so that all people can be cared for, using a unified co-operative approach of professionals and communities working together. We argue that not only is this a practical way of solving what appears to be unaffordable financial demands, it is actually preferable in terms of quality and continuity of care experience for all concerned.

Caring for people who are ageing and dying, supporting carers, and giving support to those who are suffering loss and bereavement, has been part of human social evolution. For the majority of the world, death remains a community concern. Hands on care is performed by people in the community sometimes in partnership with professional carers. Support for the carers and the bereaved too is performed by the whole community rather than isolated individuals. Poorer nations with limited access to 
healthcare services have little choice other than making death a community matter.

There has been increasing interest in public health approaches to end-of-life care since the mid-1990s, following the development of the principles of 'health promoting palliative care' by one of the authors (AK). ${ }^{5-7}$ The concise principles of this approach can be found in the compassionate city charter. ${ }^{8}$

\section{Compassionate Cities are communities that recognise that all natural cycles of sickness and health, birth and death, and love and loss occur everyday within the orbits of its institutions and regular activities. A com- passionate city is a community that recognises that care for one another at times of crisis and loss is not simply a task solely for health and social services but is everyone's responsibility.}

Compassionate Cities are communities that publicly encourage, facilitate, supports and celebrates care for one another during life's most testing moments and experiences, especially those pertaining to lifethreatening and life-limiting illness, chronic disability, frail ageing and dementia, grief and bereavement, and the trials and burdens of long term care. Though local government strives to maintain and strengthen quality services for the most fragile and vulnerable in our midst, those persons are not the limits of our experience of fragility and vulnerability. Serious personal crises of illness, dying, death and loss may visit any us, at any time during the normal course our lives. A compassionate city is a community that squarely recognises and addresses this social fact.

Compassionate communities can be built wherever there are people, and that is almost everywhere. Initiatives can take place in schools, workplaces, trade union movements, hospices, care homes, neighbourhoods and high streets, social media and educational institutions. This can be supported by policies from local, regional and national government and encouraged in a variety of ways, including public recognition of the reality of the experiences of death, dying and loss through exhibitions, prizes and annual public ceremonies such as parades. The compassionate city charter is something of a checklist of the different areas that can be addressed. The principles of public health approaches can be applied the world over and many examples of this were presented at the recent 4th Public Health Palliative Care Conference in Bristol in 2015 and in the recent edited volume by Wegleitner et $a l^{9}$ Solutions about how to look after people at end of life, their carers and the bereaved are developed locally and worked out at ground level by the people who are involved.

\section{PRACTICAL APPLICATION OF PUBLIC HEALTH APPROACHES}

A central issue for public health approaches to end-of-life care is how we can make the most out of community assets and capacity, enabling the dying to be supported by the people who are closest to them. There are a variety of ways of doing this. The following are typical illustrations:

- Network development. Naturally occurring supportive networks surround both patients and carers. There are a variety of examples of how networks can be stimulated, organised and developed. For example, a very common experience is that when support is offered, particularly during the early stages of an illness, this is turned down. Both patients and their carers do not want to be a burden to those around them. As offers of help are turned away, people become increasingly isolated. This becomes more problematic as people become less well, when more support is needed. A very simple principle of network development is to always just say yes whenever help is offered. This helps networks to become activated and developed at the early stages of an illness. Networks can also be developed directly by asking and organising. This is a skill that takes time to learn but can be supported and taught, using well established principles of community development.

- Development of supportive communities of volunteers. Befriending schemes can be supportive in a wide variety of different ways. This might be support given to carers in the daily tasks that are needed to run a household, such as cooking, cleaning, shopping or caring for pets. It might be emotional support for a carer by offering a listening ear, or even making life normal by doing the normal things such as going out for a meal, see a film or go for a drink. It can also be offering direct support to the person with the illness. There is a tendency to think that hands on care is the domain of professionals. However, their reality of often very different with the majority of hands on care being done not by the main carer but by those in the inner network of close family and friends. Direct support can also involve tasks such as lifts, spending time with someone or giving a carer some respite.

Creation of supportive policies within institutions. Institutions encompass a wide variety of different organisations. This will include educational institutions, workplaces, trade unions, schools and religious organisations to name but a few. Within an institution, a way of thinking about capacity building is to consider three different aspects.

1. Creation of compassionate policies. This is a way an institution (eg, a workplace or a school) can officially give permission, help and support those people with terminal illnesses, carers or people who have experienced grief and bereavement. Recently the BBC interviewed the brother of a worker killed in an explosion at work. The company of the worker had not approached the family before the TV crews and he expressed bitterness at their slowness. ${ }^{10}$ This is fertile ground for poor bereavement outcomes and a law suit. If the company had a policy about what to do in these kinds of situation these volatile potentialities could be avoided. 
2. Asking for volunteers for people within the institution to act as emotional support and to be a listening ear. This may be people who have a natural affinity for doing this or maybe people who have undergone the experience of loss which may be of help to others. These are internal processes that not only improve quality of life for all workers-as do antisexual harassment or antibullying policies-they also enhance company and workplace loyalty and support.

3. Having a volunteer 'bank' of people who are prepared to do tasks, such as giving lifts, covering colleagues at work, collecting children from school. The whole workforce, school or temple can participate as little or as much as they would want. This approach takes volunteering away from recreational or palliative care sites and situates these habits into the very fabric of everyday biography throughout the life course.

As can be imagined, working or studying in an environment that has a place for such kindness adds a lot to the quality of experience for all those involved. It is not uncommon to hear someone say "yes, I did know that my colleague's father had died but I didn't know what to say, so I said nothing". Being in a place where help and support is offered is very different.

\section{HOW PALLIATIVE CARE CAN BE REFRAMED TO INCLUDE EVERYONE IRRESPECTIVE OF DIAGNOSIS.}

A recent report from the University of Western Sydney $^{11}$ described the type of networks and quality of relationships surrounding people who died at home. The report examines in detail how professional services struggle to interact with these nonprofessional networks. We believe that network development and community capacity building offers a way to widening the access to palliative care and increasing the support given to people at end of life.

Thinking about reframing palliative care can be aided by asking two further questions. First, what work currently carried out by professionals could be performed by communities? This is a challenging question because professionals find so much meaning and value in what they do. Thinking that they may not be the best person to help can cause anxiety about their role and place. Second, if the community served by the professional was a 3 hour journey to their closest service, what solutions would the professional come up with to provide care? A concern for many professionals is that the quality of care diminishes when performed by non-professionals. This is not our experience and if training is needed, it can be organised. Further studies will be needed to assess the impact of the use of non-professionals on care quality.

Finding answers to these two questions helps to identify how we can make best use of the limited professional resources of formal and professionally-led palliative care. If we can do this, we stand a chance of reconfiguring services to meet the needs of all.
De-professionalisation of care can be thought about in the following areas

- Psychological and social care. These might include advance care planning, organised neighbourhood assistance and bereavement support. People who have been through the experience of being a carer can offer significant support and benefit to those going through the same, by helping them navigate their way through the variety of expected problems. For carer or patient, knowing that someone else understands because they have been a carer themselves makes talking much easier. It also assists in the removal of some of the patient-professional barriers. There are a variety of models for this, with terms such as 'compassionate neighbours' or 'community companions' often used. We have found that both the people who are given support and the volunteers themselves say that the relationships formed offer support and benefit in a way that cannot be given by professionals. A central focus that seems to help people is to know that the experiences that they are undergoing are normal human ones This commonality of experience helps to lessen the extreme emotion swings of what appears as frightening, difficult or mysterious events.

- Physical care. Hands on care, such as cleaning, washing and dressing is seen as a professional issue when the caring network is stretched. There are other solutions to providing hands on care, which include training and enabling the caring network, finding an increased network of people who are willing to do this and by supporting community groups of volunteers who can provide hands on care.

- Redefining the role of specialist palliative care nurses. Some of their work does not require specialist input. There are a wide variety of these tasks which can be carried out by non-specialist or volunteer. For example, arranging prescription and collection of medication via general practice surgeries can be very time consuming. Likewise making sure just in case medications at end of life are in the house can determine whether the dying person is able to stay at home or be admitted to hospital as an emergency. Another cause of significant anxiety and suffering for patients and relatives is the delay in administration of symptom relieving injections while waiting for a health professional to attend the house. Interestingly, the National Survey of Bereaved People (Voices) $^{12}$ highlights that pain and other symptom control at home is not as good as in institutions. It is common for people to wait a number of hours for a health professional to visit. A simple solution for some people is to train relatives to deliver subcutaneous medication. This is a solution that is found all over the world and protocols which involve relatives and pharmacists are well established. It is however not common practice, particularly in the UK

- Implementation of the compassionate city charter (8). Death and dying is everyone's business. It takes a village to raise a child and it takes a community to support death, dying and bereavement. Involving everyone, 
in terms of engagement and in giving help, is a rich resource for finding volunteers who are willing to provide practical support. It is important to raise the bar on community involvement and the charter encourages local government to play an active role as change agent in the policy development of schools, workplaces, commercial districts or churches/temples under its jurisdiction as well as providing awards and festivals of recognition of the importance of supportive care in tough personal times.

\section{A NEW MODEL OF CARE}

Our proposed model of care is one where palliative care and community support work together in partnership. Much of the work currently carried out by palliative care professionals can be performed by working with communities and volunteers. Our experience is that when this happens, the experiences are more meaningful to both caregivers and patients alike. Decreasing the current workload of palliative care professionals offers the opportunity for service redesign. Identification of people with life-limiting illness is easier for professionals when they know that there is something extra that can be done to improve the quality of their remaining life. A very successful example of this can be found in the project developed and run by Paul Cronin at Severn Hospice, ${ }^{13}$ where GP surgeries were able to identify with ease their 35 most vulnerable, frail patients. This is already nearly half of the people who are likely to die within a year for an average GP practice size of 10000 patients.

Freeing up workforce then gives the possibility of extending services to all people who have terminal illnesses irrespective of diagnosis. We envisage teams of carers, families, friends, neighbours and volunteers working directly in localities with palliative care nurses to provide support in multiple ways for the last phases of life. Health and social care professionals are also part of this team. The freeing of resources means that the most vulnerable and those most at need can use the limited availability of professional caring services. It is uncertain as to the scale of the impacts of using the variety of practical examples of a public health approach and evaluation and research will be an important facet of any service changes.

\section{SUMMARY}

The focus of specialist palliative care on mainly patients with cancer can be seen as part of the continuous development of palliative care services. We believe that maintaining this position is an inequity and an historical anachronism. The current reality is that people need support irrespective of diagnosis and the inequity we now see in palliative care is a fundamental matter of social justice. Furthermore, the confinement of our attentions to medical and nursing workforce demands within palliative care is a major retreat from the basic vision of palliative care as a promise of physical, psychological, social and spiritual care delivery at the end of life. Moreover, this narrowing of priorities is at the same time a failure of our capacity to use public health models, models that have proven to be effective in other health service areas ${ }^{14}$ as well as end-of-life care, ${ }^{15}$ to shore up the deficits in our traditional ways of offering social support at the end of life.

Hospices are ideally placed to lead the change in how to support people at end of life, but they are not exclusively so. Hospices are accustomed to working with volunteers. They work in people's homes at end of life and often work as a primary source of communication for coordinated end-of-life care. The model we have proposed employs teams of naturally occurring supportive networks enhanced by making best use of families, friends, neighbours, local government and businesses, as well as community members and supported by health and social care professionals looking after people in their communities. We do well to remember that death, dying, caregiving and loss are social problems with medical aspects to them and not medical problems with social aspects. Viewed in this way, it is crucial that we seek social solutions to make the experience as meaningful and supportive as possible.

Contributors Dr Abel and Professor Kellehear are joint authors. Competing interests None declared.

Provenance and peer review Not commissioned; externally peer reviewed.

\section{REFERENCES}

1 IQ N. Archived content National End of Life Care Programme, 2014 (cited 5 May 2015). http://webarchive.nationalarchives. gov.uk/20130718121128/http:/endoflifecare.nhs.uk

2 Quality NI. Economic Evaluation of the Electronic Palliative Care Coordination System (EPaCCS) Early Implementer Sites NHS IQ2013 (cited 5 August 2015). NHS IQ Report]. http:// www.england.nhs.uk/wp-content/uploads/2013/05/ economic-eval-epaccs.pdf

3 A J. 9 out of 10 councils forced to cut social care access localgovernmentexecutive.co.uk2014 (cited 6 August 2015). http://www.localgovernmentexecutive.co.uk/news/ 9-out-10-councils-forced-cut-social-care-access

4 EOL Profiles: Cause and Place of Death-CCG [Internet]. (cited 18 December 2015). http://www. endoflifecare-intelligence.org.uk/profiles/CCGs/Place_and_ Cause_of_Death/atlas.html

5 Kellehear A. Health-promoting palliative care: developing a social model for practice. Mortality 1999;4:75-82.

6 Haraldsdottir E, Clark P, Murray SA. SERVICE IMPROVEMENT-Health-promoting palliative care arrives in Scotland. Eur J Palliat Care 2010;17:130.

7 Paul S, Sallnow L. Public health approaches to end-of-life care in the UK: an online survey of palliative care services. BMJ Support Palliat Care 2013;3:196-9.

8 Kellehear A, Wegleitner K, Heimerl K. The compassionate city charter IN compassionate communities: case studies from Britain and Europe. Abingdon, UK: Routledge, 2015.

9 Wegleitner K, Heimerl K, Kellehear A. Compassionate communities: case studies from Britain and Europe. Routledge, 2015. 


\section{Feature}

10 News B. Bosley Mill explosion: 'Disastaer waiting to happen'. BBC news. 2015.

11 Horsfall D, Yardley A, Leonard R, et al. End of Life at home Co-creating an ecology of care. Australia: University of Western Sydney, 2015 May 2015. Report No.

12 Statistics OoN. National Survey of Bereaved People (VOICES) by NHS Area Team (England), 2012 to 2013. Office for National Statistics. 26 March 2015. Report No.
13 Klaus Wegleitner KH, Kellehear A. Compassionate communities: case studies from Britain and Europe. UK 2015 06/07/2015. $222 \mathrm{p}$.

14 O’Mara-Eves A, Brunton G, McDaid G, et al. Community engagement to reduce inequalities in health: a systematic review, meta-analysis and economic analysis. Public Health Res 2013;1.

15 Sallnow L, Richardson H, Murray SA, et al. The impact of a new public health approach to end-of-life care: a systematic review. Palliat Med 2015. 